Published in Astrophysical Journal Letters

Preprint typeset using $\mathrm{IAT}_{\mathrm{E}} \mathrm{X}$ style emulateapj v. 08/22/09

\title{
STELLAR KINEMATIC CONSTRAINTS ON GALACTIC STRUCTURE MODELS REVISITED: BAR AND SPIRAL ARM RESONANCES
}

\author{
T. Antoja ${ }^{1}$, O. Valenzuela ${ }^{2}$, B. Pichardo ${ }^{2}$, E. Moreno ${ }^{2}$, F. Figueras $^{1}$, D. Fernández $^{1}$ \\ ${ }^{1}$ Institut de Ciències del Cosmos, IEEC-UB, Universitat de Barcelona, Martí i Franquès, 1, E-08028 Barcelona, Spain; tantoja@am.ub.es \\ ${ }^{2}$ Instituto de Astronomía, Universidad Nacional Autónoma de México, A.P. 70-264, 04510, México, D.F.; octavio@astroscu.unam.mx \\ Published in Astrophysical Journal Letters
}

\begin{abstract}
We study the phase space available to the local stellar distribution using a Galactic potential consistent with several recent observational constraints. We find that the induced phase space structure has several observable consequences. The spiral arm contribution to the kinematic structure in the solar neighborhood may be as important as the one produced by the Galactic bar. We suggest that some of the stellar kinematic groups in the solar neighborhood, like the Hercules structure and the kinematic branches, can be created by the dynamical resonances of self-gravitating spiral arms and not exclusively by the Galactic bar. A structure coincident with the Arcturus kinematic group is developed when a hot stellar disk population is considered, which introduces a new perspective on the interpretation of its extragalactic origin. A bar-related resonant mechanism can modify this kinematic structure. We show that particles in the dark matter disk-like structure predicted by recent LCDM galaxy formation experiments, with similar kinematics to the thick disk, are affected by the same resonances, developing phase space structures or dark kinematic groups that are independent of the Galaxy assembly history and substructure abundance. We discuss the possibility of using the stellar phase space groups as constraints to non-axisymmetric models of the Milky Way structure.
\end{abstract}

Subject headings: dark matter — Galaxy: disk - Galaxy: evolution - Galaxy: kinematics and dynamics - Galaxy: structure — solar neighborhood

\section{INTRODUCTION}

The study of stellar kinematic groups in the solar neighborhood has a long tradition in Galactic astronomy, going back to the discovery of the Hyades and Ursa Major groups (Proctor 1869). In Antoja et al. (2008) we presented a study of the solar neighborhood kinematic groups using a sample of 24,190 stars. We confirmed the existence of the Sirius, Coma Berenices, Hyades-Pleiades and Hercules branches (Fig. 1 $\mathrm{k}$ ). They all have a negative slope of $\sim 16^{\circ}$ in the $U-V$ plane (following the standard definitions of $U$ and $V$ ) and a slight curvature.

Considerable amount of work has been performed in an attempt to explain the origin of these kinematic groups. External processes like past accretion events (Navarro et al. 2004) and internal disk processes like star formation bursts or secular dynamics have been proposed. Although they were initially considered mutually exclusive, all such mechanisms are natural in current galaxy formation models (Klvpin et al. 2008; Romano-Díaz et al. 2008; Ceverino \& Klypin 2007b). Recently, the hypothesis of the disk-dynamical origin of these structures has gained popularity partially because of the consistency of the Hercules structure with the effects of the Galactic bar resonances (Kalnais 1991; Dehnen 2000; Fux 2001). In addition, steady or transient spiral arms were proposed to explain the characteristics of some of these stellar groups (Skulian et al. 1999; De Simone et al. 2004; Famaey et al. 2005; Quillen \& Minchev 2005). A more recent study argues that the combined effect of a bar and spiral arms is necessary to accurately reproduce observations in the solar neighborhood (Chakrabartv 2007). In that case, spiral arms at the weaker end of the observa- tional range (with a fractional amplitude of less than $4 \%$ of the background disk density) were used. However, in that study the spiral arms seem to contribute only in the fine structure, thus weakening constraints on the Milky Way (MW) spiral structure based on the solar neighborhood kinematics. Previous studies have considered models for the non-axisymmetric components of the Galaxy motivated by the dynamical point of view, in particular weak spiral arms described by a cosine function and bars described by the quadrupole perturbation. However, it is unclear whether there is any dependence of the induced local solar neighborhood kinematics on the detailed Galactic structure. Moreover, the initial conditions hardly consider the evolution of the MW.

Our contribution improves upon earlier studies. First we investigate the stellar kinematic response to a model that satisfies published observational constraints to the MW structure, in particular to the non-axisymmetric components. Secondly, we consider for the first time initial conditions and integration times that attempt to represent stars born at different times and with different kinematic conditions, like those in the solar neighborhood. Lastly, we investigate effects on the local dark matter kinematics, in particular in the disk-like dark matter structure recently predicted by LCDM models. This issue is important to predict signals in direct dark matter detection experiments.

\section{SIMULATIONS}

In order to study the effect of the non-axisymmetric Galactic structure on the solar neighborhood kinematic distribution, we have performed numerical integrations of test particle orbits on the Galactic plane, adopting the initial conditions discussed in Sect. 2.2 and the po- 
TABLE 1

Non-Axisymmetric Galactic Disk Components (PICHARDO ET AL. 2003, 2004)

\begin{tabular}{lc}
\hline \hline \multicolumn{1}{c}{ Parameter } & Value \\
\hline Bar axis ratio & $10: 3.12$ \\
Bar scale lengths $(\mathrm{kpc})$ & 1.7 and 0.54 \\
Bar angle respect to the Sun $\left(^{\circ}\right)$ & 20 \\
Bar mass $\left(\mathrm{M}_{\odot}\right)$ & $10^{10}$ \\
Bar pattern speed (see text) $\Omega_{b}\left(\mathrm{~km} \mathrm{~s}^{-1} \mathrm{kpc}^{-1}\right)$ & 60,45 \\
Spiral arm pitch angle $\left({ }^{\circ}\right)$ & 15.5 \\
Spiral arm scale-length $(\mathrm{kpc})$ & 2.5 \\
Spiral arm mean force ratio $(\%)$ & 10 \\
Spiral arm pattern speed $\Omega_{s p}\left(\mathrm{~km} \mathrm{~s}^{-1} \mathrm{kpc}^{-1}\right)$ & 20 \\
\hline
\end{tabular}

tential described in Sect. 2.1. Each particle integration time is initialized at a value $t=-\tau$ and ended at $\mathrm{t}=0$, where $\tau$ is the time during which the particle is exposed to the non-axisymmetric potential. The induced kinematic distribution at the end of the simulation is studied by considering the particles inside a circle of radius $500 \mathrm{pc}$ centered at the solar position. Finally, the predicted and the observed distributions are compared. The motion equations were integrated with the Bulirsch-Stoer algorithm of Press et al. (2004), conserving Jacobi's integral within a relative variation of $\left|\left(E_{J i}-E_{J f}\right) / E_{J i}\right| \approx 10^{-11}$ for only-arms and only-bar models. The reference frame used for the calculations is the rotation frame of the spiral arms when only this non-axisymmetric component is considered and the one of the bar in the other cases. In all cases, we check that the number of particles in the final distributions is statistically robust.

\subsection{The Galactic Model}

We use the Galactic potential described by Pichardo et al. (2003, 2004) consistent with several recent observational constraints. For our study the most relevant parts are the bar and spiral arm components, whose corresponding parameters are presented in Table [1. The model is compared with observations and with other models elsewhere (Pichardo et al. 2003, 2004). The bar consists of a prolate mass distribution that resembles Model S of Freudenreich (1998) from COBE/DIRBE data. We consider two alternative bar pattern speeds (60 and $45 \mathrm{~km} \mathrm{~s}^{-1} \mathrm{kpc}^{-1}$ ) according to the range found by Debattista et al. (2002). The spiral arm model is a $3 \mathrm{D}$ steady model with two arms that traces the locus reported by Drimmel \& Spergel (2001) using K-band observations, and also in agreement with the latest results from Spitzer (Benjamin 2009, private communication). The solar radius is close to the bar outer Lindblad resonance (OLR) and to 4:1 inner Lindblad resonance (ILR) of the spiral arms. The ratio of the radial force of the spiral arms to the background peaks at $\sim 1.5: 10$ and the mean value along the arm is approximately 1:10 (see Pichardo et al. 2003). This is at the higher end of the limits suggested by Patsis et al. (1991) for MW type galaxies. Instead of introducing the spiral arms and bar adiabatically to avoid transient features on the model, we prefer to test self-consistency through an analysis of the stellar orbital reinforcement of the potential, as in Patsis et al. (1991), presented in Pichardo et al. (2003, 2004).

\subsection{Initial Conditions}

The choice of appropriate initial conditions is somewhat controversial. Traditionally they are motivated by self-consistency with the present stage of the Galactic disk structure. However, current scenarios of galaxy formation and evolution predict that both external and internal perturbation mechanisms affect the disk kinematics. The integration time in our simulations is intended to consider only the latest stages in the Galaxy evolution. This late evolution was affected or even dominated by secular dynamical processes (Klypin et al. 2008). We avoided longer integration times (more than 2 Gyr) because they might require the inclusion of Galactic evolution like bar weakening or transient arms, as well as the effect of external perturbations to the MW disk, thus masking the effect of the current MW structure. Therefore we focused on the recently induced kinematic structure in the solar neighborhood. We explored three different types of initial conditions (IC1, IC2, IC3), all of them assuming an exponential disk scale length with $R_{\rho}=2.5 \mathrm{kpc}$.

IC1 - The initial velocity distribution relative to the Regional Standard of Rest is adopted as a Gaussian (with $\sigma_{U}=\sigma_{V}=5 \mathrm{~km} \mathrm{~s}^{-1}$ ) constant for all radii. Each particle is exposed to the non-axisymmetric perturbations for a time $(\tau)$ chosen at random between 0 and 2 Gyr. This maximum integration time corresponds approximately to 14-20 and 6 revolutions of the bar and the spiral arms, respectively. With this set of initial conditions we aim to simulate the birth of stars in the disk with small velocity dispersion and the effect of having stars with different ages (different integration time) at the solar position ${ }^{1}$.

IC2 - The distribution function satisfies the collisionless Boltzmann equation as it is discussed in Hernquist (1993). The velocity dispersion has an exponential profile with scale length $R_{\sigma}=7.5 \mathrm{kpc}$ and local normalization $\sigma\left(R_{\odot}=8.5 \mathrm{kpc}\right) \sim 20 \mathrm{~km} \mathrm{~s}^{-1}$. In this case the integration time is fixed at $\tau=400 \mathrm{Myr}$ for all particles (corresponding approximately to 3-4 bar revolutions and similar to the value of Dehnen $(2000))$. With these initial conditions, we can study the relatively rapid induced effects of the non-axisymmetric component on the local kinematics.

IC3 - Identical to IC2 but with a higher velocity dispersion of $\sigma\left(R_{\odot}=8.5 \mathrm{kpc}\right) \sim 40 \mathrm{~km} \mathrm{~s}^{-1}$, closer to an old thin disk component, or to the thick disk. Recent studies of the formation of MW mass galaxies predict that a flattened dark matter structure mirroring the thick disk properties will form in a LCDM Universe (Bruch et al. 2008; Read et al. 2008). Therefore, these initial conditions are also consistent with particles in the dark disk.

\section{SPIRAL ARM AND BAR CONTRIBUTION TO THE LOCAL KINEMATIC STRUCTURE}

Models Using Only Spiral Arms - In this section we present the kinematic structure developed by the selfgravitating spiral arm model. We find that this model produces ample substructure. In particular, assuming

1 N-body disk simulations assuming a low Q parameter (e.g. Thomasson et al. 1991) show that a configuration with strong spiral arms and low velocity dispersion can be sustained for at least 7 disk revolutions. These experiments are similar to our integrations using IC1. 

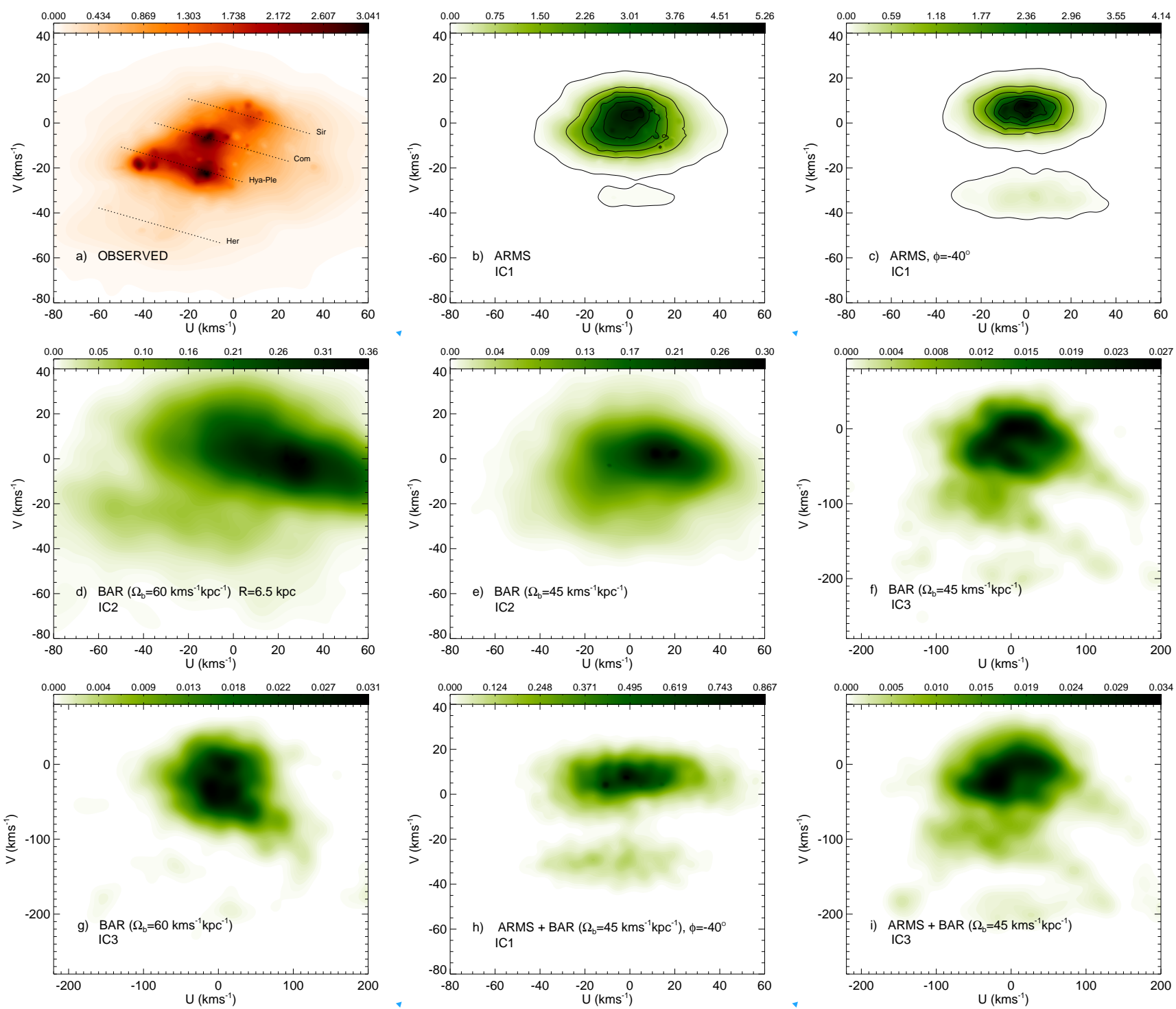

Fig. 1. - Density field in the $U-V$ plane obtained by wavelet denoising for: a) the observed sample; b, c) simulations with a model using only spiral arms; d, e, f, g) simulations with a model using only bar; h, i) simulations with a combined model (bar + spiral arms). Notice the different scales of the panels.

low velocity dispersions (IC1), it reproduces a branch at low angular momentum (with $V \sim-40 \mathrm{~km} \mathrm{~s}^{-1}$ consistent with the Hercules structure) separated from a central group of substructures. To our knowledge, it is the first time that an unbarred model has produced a similar structure. Figure 10 shows the $U-V$ plane for the solar position. Although the shape of the structure at $V=-40 \mathrm{kms}^{-1}$ is not exactly equal to the Hercules branch, it does show that the spiral arms by themselves crowd the velocity space at these negative $V$. In particular, the test particles in this kinematic group have a perturbation exposure time of at least $\sim 1$ Gyr. The central part of the distribution seems to be split into two groups or branches, resembling some of the observed central kinematic branches (Fig. 19). In order to explore small variations respect to the configuration of Drimmel \& Spergel (2001), given the uncertainty on the exact position of the arms, we also show in Figure 1k the $U-V$ plane for a region located at $\phi=-40^{\circ}$, that is at the solar circle but at $40^{\circ}$ of the Sun in the counter- clockwise direction. In this case and for a wide range of $\phi$, the equivalent to the Hercules structure is also generated. We conclude that the contribution of the spiral arms to the solar neighborhood kinematics may be comparable to that of the bar. The sensitivity of our results to the properties of the arms indicates that local kinematics can be used as one of the constraints to the current observational ambiguities about this non-axisymmetric component.

Models Using Only a Bar - In agreement with previous studies (e.g. Dehnen 2000), we find that a Galactic model using only a bar can trigger a kinematic group at negative $V$ and $U$ resembling the Hercules branch. However, in our experiments this branch can be populated only under some combinations of model parameters and initial conditions. For example, a bar pattern speed of $\Omega_{b}=60 \mathrm{~km} \mathrm{~s}^{-1} \mathrm{kpc}^{-1}\left(R_{O L R} / R=0.74\right)$ under IC2 produces a branch at an incorrect position $(U \geq 0)$. However, if the imaginary solar observer is located at an inner 
radius $R=6-7 \mathrm{kpc}$ (where $R_{O L R} / R=0.97$ ) a structure appears at $V \sim-35 \mathrm{~km} \mathrm{~s}^{-1}$ and $U \leq 0$, as is shown at the top left of Figure 1 $\mathrm{d}$. A kinematic group at $V$, $U \leq 0$ also appears if the bar pattern speed is reduced to $45 \mathrm{~km} \mathrm{~s}^{-1} \mathrm{kpc}^{-1}$ (Fig. 1e), although the mean branch velocity $V$ is now slightly smaller: $V \sim-20 \mathrm{~km} \mathrm{~s}^{-1}$ (again with $\left.R_{O L R} / R=0.97\right)$. In summary, the Hercules branch at the correct position may be achieved by varying either the model pattern speed, the observer position or even the initial conditions.

If we consider hotter initial conditions (IC3), archshaped structures appear at lower $V$. These structures appear even if we consider an axisymmetric disk model, as also recently reported by Minchev et al. (2009) who assumed a non-relaxed model. In our simulations the positions of these kinematic arches are modified when the bar is added to the model. This suggests that both kinematic initial conditions and Galactic structure contribute to create the kinematic groups. For example, differences are observed if we use either $\Omega_{b}=45 \mathrm{~km} \mathrm{~s}^{-1} \mathrm{kpc}^{-1}$ (Fig. 1f) or $\Omega_{b}=60 \mathrm{~km} \mathrm{~s}^{-1} \mathrm{kpc}^{-1}$ (Fig. 1 $\mathrm{s}$ ). The arches developed at $V \sim-40 \mathrm{~km} \mathrm{~s}^{-1}$ and $-100 \mathrm{~km} \mathrm{~s}^{-1}$ for the lower bar pattern speed (not seen when $\Omega_{b}=60 \mathrm{~km} \mathrm{~s}^{-1} \mathrm{kpc}^{-1}$ ) are closer to the Hercules and Arcturus observed structures. ${ }^{2}$ This result should be considered only as evidence of sensitivity to the model but not necessarily as favoring a particular $\Omega_{b}$ value. Furthermore, these simulations show the important role of the bar in the development of the local kinematic structure.

Combined Models - Here we present the results obtained with the model that includes both the bar and the spiral arm perturbations. This model, with different pattern speeds for the bar and the spirals, is supported by recent studies like Patsis et al. (2009). They reported that the best match between observations and models for the galaxy NGC 3359 is found when different pattern speed for the bar $\left(39 \mathrm{~km} \mathrm{~s}^{-1} \mathrm{kpc}^{-1}\right)$ and for the spiral arms $\left(15 \mathrm{~km} \mathrm{~s}^{-1} \mathrm{kpc}^{-1}\right)$ is considered. Now we show examples of how the branches generated by the spiral arms or the bar are maintained when the combined model is used. Figure 1h shows the results for the spiral arms plus the bar with $\Omega_{b}=45 \mathrm{~km} \mathrm{~s}^{-1} \mathrm{kpc}^{-1}$ used with IC1 near the solar position $\left(\phi=-40^{\circ}\right)$. In Figure 11, we show the case corresponding to spiral arms and a bar with $\Omega_{b}=45 \mathrm{~km} \mathrm{~s}^{-1} \mathrm{kpc}^{-1}$ under IC3. Here the arch-shaped structures associated with Hercules and Arcturus are still observed.

The diversity of the initial conditions in our simulations is not extensive. Moreover, the observed velocity field is likely to be the result of the combined action of several Galactic processes. Therefore, comparison between the observed velocity distribution ${ }^{2}$ (Fig. 1a) and the results of test particle simulations is not straightforward. A first approach might be to compare the observed velocity field with the combined model results under IC1 and IC3, where the central branches and the kinematic

\footnotetext{
2 Notice that the observed Arcturus structure is out of the range of Figure 17a as this structure is not prominent in the sample used by Antoia et al. (2008). See Figure 2 of Williams et al. (2008) for a $U-V$ plane containing the Arcturus structure.
}

groups at low angular momentum would appear simultaneously. The next complexity level might require the inclusion of age and kinematic criteria in the definition of the artificial observational sample. We defer such details to a future study, since they do not alter the conclusions of this Letter.

\section{LOCAL DARK MATTER KINEMATICS}

Another unexpected aspect of the bar- and spiral arminduced phase space structure is the effect on the local dark matter kinematics. Particles in a possible dark matter bar (Colín et al. 2006), dark disk (Bruch et al. 2008; Read et al. 2008) and even in the dark matter halo (Athanassoula 2005) are trapped/scattered in the same resonances as stars are. It has been shown that different bar-induced resonances can be populated by disk-like and halo particles (Athanassoula 2002, 2003; Ceverino \& Klypin 2007).

Our study considers only the case of the dark disk. Following (Read et al. 2009) we assume that our IC3 initial conditions set also represents the dark particles in the dark thick disk. Thus Figures 1f, 15 and 10, obtained from IC3, also reproduce the local dark matter kinematics induced by our MW dynamical models. Our results show that these models generate dark matter currents inside the Galactic dark disk. These dark-matter currents would be independent of the Galactic assembly history or the dark substructure abundance. The dynamical history of the Galaxy and its detailed large scale structure may help to establish whether the amplitude of the dark matter kinematic structure is detectable by planned dark matter detection experiments.

\section{DISCUSSION AND CONCLUSIONS}

The orbital analysis of a MW model consistent with published observational constraints has several unexpected consequences for the local stellar kinematics.

The spiral arm contribution to the resonant structure in the solar neighborhood may be comparable to that of the Galactic bar. The main differences to previous studies are the arm force contrast and force field shape (Pichardo et al. 2003, Fig. 5, 8 and 9), as well as the variety in initial conditions. In particular, we find that the Hercules structure may be produced by the spiral arms and not exclusively by bar resonances as traditionally believed. Dehnen (2000) concluded that the Hercules branch is unlikely to have been produced by resonant scattering processes due to spiral arms. His main argument is that spiral arms do not act on stars with epicycles greater than the interarm separation. However, the epicycle amplitude of the stars in the structure created in our simulations ${ }^{3}$ (at $U \sim[-30,30]$ and $V \sim[-45,-35]$ ) is about $3 \mathrm{kpc}$. On the other hand, the interarm separation in the different loci of our model is in the range $5.5-7 \mathrm{kpc}$. This is sufficient to produce the Hercules structure and still in agreement with Dehnen (2000). Other authors like Quillen \& Minchev (2005) reproduced the Hyades-Pleiades and Coma Berenices branches as ascribed to periodic orbits related to the spiral arm 4:1 ILR, but not the Hercules structure. For some parameter combinations our model reproduces both kinds of structure.

\footnotetext{
3 This value is obtained theoretically from the epicycle approxi-
} mation (e.g. Asiain et al. 1999) or directly from orbital integration. 
Detailed comparison with other authors is not straightforward because many of them used the tight-winding approximation to model the spiral arms, a different simulation strategy, and in some cases, even a four-armed model (Chakrabarty 2007), while ours includes two arms.

As in previous studies, we confirm that the Hercules structure can also be produced by the Galactic bar under certain combinations of the model parameters and initial conditions. However, we considered a prolate bar (for the detailed force field see Fig. 16 of Pichardo et al. 2004) instead of the widely used cosine bar potential (Dehnen 2000). The study of possible differences in the local kinematic structure generated by each model is postponed for a future study.

A subset of our experiments develops a structure resembling the Arcturus kinematic group. The required condition seems to be a relatively hot stellar disk population similar to the thick disk in kinematics. This kinematic group may have arisen from a past accretion event (Navarro et al. 2004; Villalobos \& Helmi 2009). Alternatively Williams et al. (2008) recently discussed a possible disk-dynamical origin of Arcturus based on stellar population evidence, and postulated the bar 6:1 OLR as the triggering mechanism. On the other hand, Minchev et al. (2009) proposed an origin related to nonequilibrium initial conditions. Our results support an internal disk origin and, in addition, we find that the dynamics of the bar has an influence on these low angular momentum kinematic groups as it modifies these structures generated in the axisymmetric model. Preliminary results of orbit integration in 3-D show that the main arches generated in the $2-\mathrm{D}$ case are maintained and the Galactic bar significantly takes part in defining their shape. However, the 3 -D case deserves special attention and is the subject of ongoing studies. The fact that a vrms of $40 \mathrm{~km} \mathrm{~s}^{-1}$ is high enough to allow the creation of these arches in our experiments is encouraging, but it is still not sufficient to disentangle the origin of Arcturus.
A deeper discussion of this point is beyond of the scope of this letter, but it is the subject of an upcoming study.

We show that the Galactic non-axisymmetric potential develops dark kinematic groups in the dark disk predicted in cosmological simulations of galaxy formation. These currents are independent of the halo substructure abundance and may have new consequences for planned dark matter direct detection experiments.

The dependence of the stellar kinematics in the solar neighborhood on the structure, dynamics and initial conditions of our experiments suggests that kinematic groups may provide a useful constraint on nonaxisymmetric MW models. A systematic scan of the associated parameter space will be required in order to disentangle the origin of the different kinematic groups in the solar neighborhood. We are currently exploring strategies to do so. Studies like the ones discussed in this letter will derive benefit from upcoming surveys like GAIA and SEGUE2. In summary, the imprints of the non-axisymmetric Galactic structure on the local stellar kinematics are strong.

We thank G. Lake and D. Ceverino for enlightening discussions about dark matter in the MW, K. Freeman for suggesting the study of Arcturus in our simulations, and J. González, V. Avila-Reese, L. Aguilar, and H. Throop for a careful reading of the manuscript. We also thank the referee for the very helpful comments and suggestions which much improved the first version of this paper. This study was supported by MEC contracts ESP2006-13855C02-01 and AYA2006-15623-C02-02, grants CONACyT 60354 and 50720, and UNAM PAPIIT IN1 19708. T.A. was supported by the Predoctoral Fellowship of the Generalitat de Catalunya 2008FIC 00121 and by the LENAC mobility program. The simulations were run at the $\mathrm{HP}$ CP 4000 cluster (KanBalam) in the DGSCA/UNAM.

\section{REFERENCES}

Antoja, T., Figueras, F., Fernández, D., Torra, J. 2008, A\&A, 490,135

Asiain, R., Figueras, F., Torra, J. 1999, A\&A, 350, 434

Athanassoula, E. 2002, ApJ, 569, 83

Athanassoula, E. 2003, MNRAS, 341, 1179

Athanassoula, E. 2005, New York Academy Sciences Annals, 1045,168

Bruch, T., Read, J., Baudis, L., \& Lake, G. 2008, ApJ, 696, 920

Ceverino, D. \& Klypin, A. 2007, MNRAS, 379, 1155

Ceverino, D. \& Klypin, A. 2007b, ApJ, 695, 292

Chakrabarty, D. 2007, A\&A, 467, 145

Colín, P., Valenzuela, O., \& Klypin, A. 2006, ApJ, 644, 687

Debattista, V. P., Gerhard, O., \& Sevenster, M. N. 2002, MNRAS, 334, 355

Dehnen, W. 2000, AJ, 119, 800

De Simone, R.S., Wu, X., Tremaine, S. 2004, MNRAS, 350, 627

Drimmel, R., Spergel, D.N. 2001, ApJ, 556, 181

Famaey, B., Jorissen, A., Luri, X., et al. 2005, A\&A, 430, 165

Freudenreich, H. T. 1998, ApJ, 492, 495

Fux, R. 2001, A\&A, 373, 511

Hernquist, L. 1993, ApJS, 86, 389

Kalnajs, A. J. 1991, Dynamics of Disk Galaxies, eds. B. Sundelius, 323

Klypin, A., Valenzuela, O., Colin, P., \& Quinn, T. 2008, arXiv:0808.3422

Minchev, I., Quillen, A. C., Williams, M., Freeman, K. C., Nordhaus, J., Siebert, A., \& Bienayme, O. 2009, MNRAS, 396, L56
Navarro, J. F., Helmi, A., \& Freeman, K. C. 2004, ApJ, 601, L43 Patsis, P. A., Contopoulos, G., \& Grosbol, P. 1991, A\&A, 243, 373 Patsis, P. A., Kaufmann, D. E., Gottesman,S. T., \& Boonyasait, V. 2009, MNRAS, 394, 142

Pichardo, B., Martos, M., Moreno, E., \& Espresate, J. 2003, ApJ, 582,230

Pichardo, B., Martos, M., \& Moreno, E. 2004, ApJ, 609, 144

Press, W. H., Teukolsky, S. A., Vetterling, W. T., \& Flannery, B. P. 1992, Numerical Recipes in Fortran 77: The Art of Scientific Computing, 2nd ed. (Cambridge: Cambridge Univ. Press)

Proctor, R. A. 1869, Royal Society of London Proceedings Series I, 18, 169

Quillen, A.C., \& Minchev, I. 2005, ApJ, 130,576

Read, J. I., Lake, G., Agertz, O., \& Debattista, V. P. 2008, MNRAS, 389, 1041

Read, J. I., Mayer, L., Brooks, A. M., Governato, F., \& Lake, G. 2009, arXiv:0902.0009

Romano-Díaz, E., Shlosman, I., Heller, C., \& Hoffman, Y. 2008, ApJ, 687, L13

Skuljan, J., Hearnshaw, J. B., Cottrell, P. L. 1999, MNRAS, 308, 731

Thomasson, M., Donner, K.J., Elmegreen, B.G. 1991, A\&A, 250, 316

Villalobos, A., \& Helmi, A. 2009, arXiv:0902.1624

Williams, M.E.K., Freeman, K.C., Helmi, A. 2008,

arXiv:0810.2669 